\title{
Correction to: Spatial regression graph convolutional neural networks: A deep learning paradigm for spatial multivariate distributions
}

\author{
Di Zhu ${ }^{1}$ D $\cdot$ Yu Liu² ${ }^{2}$ Xin Yao ${ }^{3} \cdot$ Manfred M. Fischer ${ }^{4}$
}

(c) Springer Science+Business Media, LLC, part of Springer Nature 2022

\section{Correction to: Geoinformatica \\ https://doi.org/10.1007/s10707-021-00454-x}

Change the following sentences in the Introduction: "The commonalities and differences between SRGCNNs and linear spatial regression models are further elaborated to help understand how each component in a traditional spatial regression analysis can be formalized in the new paradigm with baseline linear models the social media check-ins data at the urban points of interest (POIs) to describe how SRGCNNs can be adopted in the urban scenario, where irregular distributed spatial multivariate data needs modeling and prediction. Furthermore, we present an alternative way to enable geographically weighted regression in SRGCNNs and compare the results with baseline linear models." to "The commonalities and differences between SRGCNNs and linear spatial regression models are further elaborated to help understand how each component in a traditional spatialregression analysis can be formalized in the new paradigm."

The original article can be found online at https://doi.org/10.1007/s10707-021-00454-x.

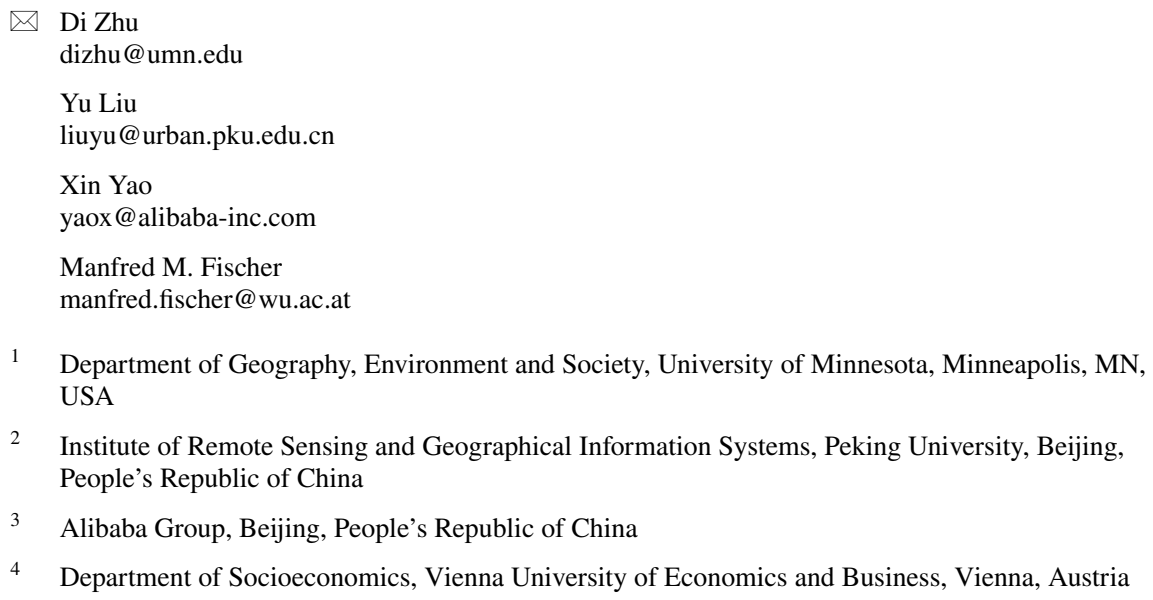

1 Department of Geography, Environment and Society, University of Minnesota, Minneapolis, MN, USA

2 Institute of Remote Sensing and Geographical Information Systems, Peking University, Beijing, People's Republic of China

3 Alibaba Group, Beijing, People's Republic of China

4 Department of Socioeconomics, Vienna University of Economics and Business, Vienna, Austria 
The acknowledgements should have read "The authors gratefully acknowledge editors, the anonymous reviewers, Dr. Tao Cheng, Dr. Yang Zhang, Dr. Ximeng Cheng, and Dr. Fan Zhang for their helpful comments. This work was partially supported by the New Faculty Set-up Funding of College of Liberal Arts, University of Minnesota (1000-10964-200425672018). Prof. Yu Liu is supported by the National Key Research and Development Program of China (2017YFB0503602) and the National Natural Science Foundation of China (41625003)."

The original article has been corrected.

Publisher's note Springer Nature remains neutral with regard to jurisdictional claims in published maps and institutional affiliations. 